\title{
PEP analysis of SDP-based non-coherent signal detection
}

\author{
Mihailo Stojnic \\ Department of Electrical Engineering \\ California Institute of Technology \\ Pasadena CA 91125, USA \\ Email:mihailo@systems.caltech.edu
}

\author{
Babak Hassibi \\ Department of Electrical Engineering \\ California Institute of Technology \\ Pasadena CA 91125, USA \\ Email: hassibi@systems.caltech.edu
}

\author{
Haris Vikalo \\ Department of Electrical Engineering \\ California Institute of Technology \\ Pasadena CA 91125, USA \\ Email: hvikalo@systems.caltech.edu
}

\begin{abstract}
In multi-antenna communication systems, channel information is often not known at the receiver. To fully exploit the bandwidth resources of the system and ensure the practical feasibility of the receiver, the channel parameters are often estimated and then employed in the design of signal detection algorithms. However, sometimes communication can occur in the environment where learning the channel coefficients becomes infeasible. In this paper we consider the problem of maximumlikelihood (ML)-detection in single-input multiple-output (SIMO) systems when the channel information is completely unavailable at the receiver and when employed signalling at the transmitter is $q$-PSK. It is well known that finding the solution to this optimization requires solving an integer maximization of a quadratic form and is, in general, an NP hard problem. To solve it, we propose an approximate algorithm based on the semidefinite program (SDP) relaxation. We derive a bound on the pairwise probability of error (PEP) of the proposed algorithm and show that, the algorithm achieves the same diversity as the exact maximum-likelihood (ML) decoder. Furthermore, we prove that in the limit of large system dimension this bound differs from the corresponding one in the exact ML case by at most $3.92 \mathrm{~dB}$ if the transmitted symbols are from 2 or 4-PSK constellations and by at most $2.55 \mathrm{~dB}$ if the transmitted symbols are from 8-PSK constellation. This suggests that the proposed algorithm requires moderate increase in the signal-to-noise ratio (SNR) in order to achieve performance comparable to that of the ML decoder but with often significantly lower complexity.
\end{abstract}

\section{INTRODUCTION}

Multi-antenna wireless communication systems are capable of providing reliable data transmission at very high rates. The channel in such systems is, in principle, unknown to the receiver and needs to be estimated either prior to or concurrently with the detection of the transmitted signal. However learning channel coefficients requires time which in environments with rapidly changing conditions can be impractical. In this paper we study the problem of ML detection when the channel information is unavailable at the receiver. The system, that we study has single transmit antenna and multiple receive antennas.

We assume a standard flat-fading channel model for multiantenna systems,

$$
X=\sqrt{\frac{\rho T}{M}} \operatorname{sh}+W
$$

where $T$ denotes the number of time intervals during which the channel remains constant, $M=1$ is the number of the transmitted antennas, $N$ is the number of the received antennas, $\rho$ is the signal-to-noise ratio (SNR), $X$ is a $T \times N$ matrix of the received symbols, $\mathrm{s}$ is a $T \times 1$ transmitted symbol vector comprised of components $s_{i}$ for which it holds that $s_{i}=\frac{1}{\sqrt{T}} e^{\frac{j 2 r \pi}{q}}, r=1, \ldots, q, q$ is an integer power of $2, H$ is an $1 \times N$ channel matrix whose components are independent, identically distributed (i.i.d.) zero-mean, unit-variance complex Gaussian random variables, and $W$ is an $N \times T$ noise matrix whose components are i.i.d. zero-mean, unit-variance complex Gaussian random variables. Furthermore, we assume that the components of $\mathbf{h}$ and $W$ are uncorrelated and that $T \geq N$, which is often the case in practice.

In the next section, we recall what the criterion for noncoherent ML-signal detection is and propose an efficient algorithm for finding its approximate solution.

\section{NON-COHERENT ML-DETECTION}

As stated in [7] the criterion for non-coherent ML-detection in system given in (1) can be written as

$$
\mathbf{s}_{\mathrm{ML}}=\arg \max _{\mathbf{s} \in \mathcal{S}} \frac{\exp \left(-\operatorname{Tr}\left\{\left[I+k \mathbf{s s}^{*}\right]^{-1} X X^{*}\right\}\right)}{\pi^{T N} \operatorname{det}^{N}\left[I+k \mathbf{s s}^{*}\right]} .
$$

where $k=\rho T$ and $\mathcal{S}=\left\{\frac{e^{\frac{j 2 \pi}{q}}}{\sqrt{T}}, \frac{e^{\frac{j 4 \pi}{q}}}{\sqrt{T}}, \ldots, \frac{1}{\sqrt{T}}\right\}^{T}$. Now, using the matrix inversion lemma and the fact that $\mathbf{s}^{*} \mathbf{s}=1$ we obtain

$$
\begin{aligned}
& \mathbf{s}_{\mathrm{ML}}=\arg \max _{\mathbf{s} \in \mathcal{S}} \frac{\exp \left(-\operatorname{Tr}\left\{\left[I-\frac{1}{k} \mathbf{s s}^{*}\right]^{-1} X X^{*}\right\}\right)}{\pi^{T N}(1+k)^{N}} \\
&=\arg \max _{\mathbf{s} \in \mathcal{S}} \operatorname{Tr}\left\{X^{*} \mathbf{s s}^{*} X\right\} .
\end{aligned}
$$

Therefore, the integer optimization problem one needs to solve can be written as

$$
\max _{\mathbf{s} \in \mathcal{S}} \operatorname{Tr}\left(X X^{*} \mathbf{s s}^{*}\right)
$$

(Since $\mathbf{s}^{*} \mathbf{s}=1$ precisely the same optimization problem is obtained if the criterion of optimization were joint channel estimation and signal detection [11]). Optimization (3) is a very difficult problem. In [8] the case $q=2$ was considered. The sphere decoder algorithm was employed to solve (3) exactly. However, for some parameters of the system, the sphere decoder may be computationally costly. In this paper, we focus on finding a computationally efficient approximate solution to (3). In particular, we relax (3) and instead solve

$$
\max _{Q \geq 0, Q_{i i}=1} \operatorname{Tr}\left(X X^{*} Q\right) \text {. }
$$


(This is a well-known semi-definite programming (SDP) relaxation, often used for obtaining approximate solutions to difficult combinatorial problems. The interested reader can find more on that in [1] and its applications in communications in excellent references [9],[10]). Let $\hat{Q}$ and $\mathrm{s}_{\mathrm{ML}}$ denote the solutions to (4) and (3), respectively. It can be shown (see [2], [3],[4]) that

$$
\alpha \operatorname{Tr}\left(X X^{*} \hat{Q}\right) \leq \operatorname{Tr}\left(X X^{*} \mathbf{s}_{\mathrm{ML}} \mathrm{s}_{\mathrm{ML}}^{*}\right)
$$

where $\alpha$ is a constant. More precisely, if $q=2$ then as shown in [2] $\alpha=\frac{2}{\pi}$, and if $q \geq 4$ then as shown in [3] and [4] $\alpha=\frac{(q \sin (\pi / q))^{2}}{4 \pi}$. Clearly, for any value $q \geq 2$ we have that $\frac{2}{\pi} \leq \alpha \leq \frac{4 \pi}{4}$.

Now, let $L$ be any lowest rank matrix such that $L L^{*}=\hat{Q}$ and $\mathbf{r}$ be a vector with zero-mean unit-variance complex Gaussian i.i.d. components. Let $\phi$ be vector of phases of components of $L \mathbf{r}$. If $\frac{2 \pi m-\pi}{q} \leq \phi_{i}<\frac{2 \pi m+\pi}{q}, 1 \leq i \leq T$, $m$ is an integer, then $\hat{\phi}_{i}=\frac{2 \pi m}{q}$. Finally let

$$
\hat{\mathbf{s}}=\frac{e^{j \hat{\phi}}}{\sqrt{T}} .
$$

Then one can write

$$
\alpha \operatorname{Tr}\left(X X^{*} \mathrm{~s}_{\mathrm{ML}} \mathrm{s}_{\mathrm{ML}}^{*}\right) \leq E_{\mid r} \operatorname{Tr}\left(X X^{*} \hat{\mathbf{s}} \hat{\mathbf{s}}^{*}\right) .
$$

Therefore, one can construct a suboptimal solution to (3) which has a guaranteed performance. Of course, strictly speaking, the performance is guaranteed only in the expected sense. However, if we repeat the randomized procedure sufficiently many times, we are very likely to obtain an instance with a cost whose value is greater than the true expectation. In fact, it was shown in [6] that, with certain modifications, the expectation in (7) can indeed be omitted. Hence, there is a polynomial time algorithm which provides a suboptimal solution to $(3), \hat{\mathrm{s}}$, such that

$$
\alpha \operatorname{Tr}\left(X X^{*} \mathrm{~s}_{\mathrm{ML}} \mathrm{s}_{\mathrm{ML}}^{*}\right) \leq \operatorname{Tr}\left(X X^{*} \hat{\mathrm{s}} \hat{\mathbf{s}}^{*}\right) .
$$

Now, in order to provide sound proofs in the following section we will slightly modify the SDP relaxation. Let $\overline{\mathrm{s}}$ be the solution of the following optimization problem

$$
\bar{s}=\arg \max _{\mathbf{s},\left.\left|\mathbf{s}^{*} \mathbf{s}\right|\right|^{2} \geq \alpha} \operatorname{Tr} X X \mathbf{s s}^{*}
$$

We refer later in the paper to this way (based on a slight modification of the standard SDP-relaxation randomized algorithm) of generating a solution $\overline{\mathrm{s}}$ as Algorithm 1 .

\section{COMPUTING THE PEP}

The probability of error can be written as

$$
P_{e}=\sum_{i=1}^{2^{T}} P\left(\text { error } \mid \mathrm{s}_{t} \text { is sent }\right) P\left(\mathrm{~s}_{t} \text { is sent }\right) .
$$

In the remainder of this section, we derive an upper bound on the $P$ (error $\mid \mathrm{s}_{t}$ is sent $)$. To facilitate this derivation, let us assume that there is a Genie who can tell us if the $\hat{s}$ found in (6) is such that $\left|\hat{\mathbf{s}}^{*} \mathbf{s}_{t}\right|^{2}<\alpha$. We formulate a slightly modified version of the Algorithm 1 and refer to it as the Genie. Its solution is $\hat{\mathrm{s}}_{1}$ such that

$$
\begin{aligned}
& \text { if } \quad\left|\hat{\mathbf{s}}^{*} \mathbf{s}_{t}\right|^{2}<\alpha \quad \hat{\mathbf{s}}_{1}=\hat{\mathbf{s}} \\
& \text { if } \quad\left|\hat{\mathbf{s}}^{*} \mathbf{s}_{t}\right|^{2} \geq \alpha \quad \hat{\mathbf{s}}_{1}=\overline{\mathbf{s}}
\end{aligned}
$$

where $\hat{\mathrm{s}}$ is as found in (6). The probability of error for the Genie algorithm is given by

$$
P_{e}^{g}=\sum_{i=1}^{2^{T}} P_{g}\left(\text { error } \mid \mathbf{s}_{t} \text { is sent }\right) P\left(\mathbf{s}_{t} \text { is sent }\right) .
$$

Clearly, our Algorithm 1 will have smaller probability of error than the Genie. They differ in the case when $\left|\hat{\mathbf{s}}^{*} \mathrm{~s}_{t}\right|^{2}<\alpha$. In that case $\hat{s}$ is incorrect and Genie keeps it as solution. On the other hand, in the same case Algorithm 1 searches for $\overline{\mathbf{s}}$ which may be solution. Therefore Algorithm 1 can work only better than the Genie. Hence, we concentrate on bounding the probability of error of the Genie, i.e., on bounding $P_{g}$ (error $\mid \mathbf{s}_{t}$ is sent). To this end, note that

$$
\begin{aligned}
& \left.P_{g} \text { (error } \mid \mathbf{s}_{t} \text { is sent }\right)=P\left(\hat{\mathbf{s}}_{1} \neq \mathbf{s}_{t}\right) \\
& \quad=P\left(\exists i: \hat{\mathbf{s}}_{1}=\mathbf{s}_{i} \neq \mathbf{s}_{t}\right) \leq \sum_{\mathbf{s}_{i} \neq \mathbf{s}_{t}} P\left(\hat{\mathbf{s}}_{1}=\mathbf{s}_{i} \neq \mathbf{s}_{t}\right) \\
& \leq \sum_{\left|\mathbf{s}_{i}^{*} \mathbf{s}_{t}\right|^{2}<\alpha} P\left(\hat{\mathbf{s}}_{1}=\mathbf{s}_{i} \neq \mathbf{s}_{t}\right)+\sum_{\left|\mathbf{s}_{i}^{*} \mathbf{s}_{t}\right|^{2} \geq \alpha} P\left(\hat{\mathbf{s}}_{1}=\mathbf{s}_{i} \neq \mathbf{s}_{t}\right) .
\end{aligned}
$$

Let us consider $P\left(\hat{\mathbf{s}}_{1}=\mathbf{s}_{i} \neq \mathbf{s}_{t},\left|\mathbf{s}_{i}^{*} \mathbf{s}_{t}\right|^{2}<\alpha\right)$ in more details. (For the brevity of notation, in the following expressions we omit that everything is conditioned on $\mathbf{s}_{t}$ being transmitted, and that $\left|\mathbf{s}_{i}^{*} \mathbf{s}_{t}\right|^{2}<\alpha$.) So,

$$
\begin{aligned}
P\left(\hat{\mathbf{s}}_{1}=\mathbf{s}_{i} \neq \mathbf{s}_{t}\right)=P\left(\hat{\mathbf{s}}_{1}\right. & \left.=\mathbf{s}_{i} \neq \mathbf{s}_{t} \mid \hat{\mathbf{s}}_{1}=\hat{\mathbf{s}}\right) P\left(\hat{\mathbf{s}}_{1}=\hat{\mathbf{s}}\right) \\
& +P\left(\hat{\mathbf{s}}_{1}=\mathbf{s}_{i} \neq \mathbf{s}_{t}, \hat{\mathbf{s}}_{1} \neq \hat{\mathbf{s}}\right) .
\end{aligned}
$$

Let us define function $C$ as $C(\mathbf{s})=\operatorname{Tr} X X^{*} \mathbf{s s}^{*}$. Furthermore, let $E$ denote the event that $\left(\hat{\mathbf{s}}_{1}=\mathbf{s}_{i} \neq \mathbf{s}_{t}, \hat{\mathbf{s}}_{1} \neq \hat{\mathrm{s}}\right)$. Clearly, $E$ implies that $C\left(\mathbf{s}_{i}\right)=C\left(\hat{\mathbf{s}}_{1}\right) \geq C(\hat{\mathbf{s}}) \geq \alpha C\left(\mathbf{s}_{t}\right)$, which further means that $C\left(\mathbf{s}_{i}\right) \geq \alpha C\left(\mathbf{s}_{t}\right)$. Using this, we obtain $P\left(\hat{\mathbf{s}}_{1}=\right.$ $\left.\mathbf{s}_{i} \neq \mathbf{s}_{t}, \hat{\mathbf{s}}_{1} \neq \hat{\mathbf{s}}\right) \leq P\left(C\left(\mathbf{s}_{i}\right) \geq \alpha C\left(\mathbf{s}_{t}\right)\right)$. Also, following similar argument, it is not difficult to see that $P\left(\hat{\mathbf{s}}_{1}=\mathbf{s}_{i} \neq\right.$ $\left.\left.\mathbf{s}_{t} \mid \hat{\mathbf{s}}_{1}=\hat{\mathbf{s}}\right) P\left(\hat{\mathbf{s}}_{1}=\hat{\mathbf{s}}\right)\right) \leq P\left(C\left(\mathbf{s}_{i}\right) \geq \alpha C\left(\mathbf{s}_{t}\right)\right)$. Replacing the obtained inequalities in (14) we have

$$
P\left(\hat{\mathbf{s}}_{1}=\mathbf{s}_{i} \neq \mathbf{s}_{t},\left|\mathbf{s}_{i}^{*} \mathbf{s}_{t}\right|^{2}<\alpha\right) \leq 2 P\left(C\left(\mathbf{s}_{i}\right) \geq \alpha C\left(\mathbf{s}_{t}\right)\right) .
$$

Now, let us consider $P\left(\hat{\mathbf{s}}_{1}=\mathbf{s}_{i} \neq \mathbf{s}_{t},\left|\mathbf{s}_{i}^{*} \mathbf{s}_{t}\right|^{2} \geq \alpha\right)$. It is easy to see that

$$
P\left(\hat{\mathbf{s}}_{1}=\mathbf{s}_{i} \neq \mathbf{s}_{t},\left|\mathbf{s}_{i}^{*} \mathbf{s}_{t}\right|^{2} \geq \alpha\right) \leq P\left(C\left(\mathbf{s}_{i}\right) \geq C\left(\mathbf{s}_{t}\right)\right) .
$$

Substituting (15) and (16) in (13), we finally obtain

$$
\begin{aligned}
P_{g}\left(\text { error } \mid \mathbf{s}_{t} \text { is sent }\right) \leq & \sum_{\left|\mathbf{s}_{i}^{*} \mathbf{s}_{t}\right|^{2} \leq \alpha} 2 P\left(C\left(\mathbf{s}_{i}\right) \geq \alpha C\left(\mathbf{s}_{t}\right)\right) \\
& +\sum_{\left|\mathbf{s}_{i}^{*} \mathbf{s}_{t}\right|^{2} \geq \alpha} P\left(C\left(\mathbf{s}_{i}\right) \geq C\left(\mathbf{s}_{t}\right)\right) .
\end{aligned}
$$


In the remainder of this section, we compute bounds on $P_{\left.i t|| \mathbf{s}_{i}^{*} \mathbf{s}_{t}\right|^{2}<\alpha}=P\left(C\left(\mathbf{s}_{i}\right) \geq \alpha C\left(\mathbf{s}_{t}\right) \mid \mathbf{s}_{t}\right.$ is sent, $\left.\left|\mathbf{s}_{i}^{*} \mathbf{s}_{t}\right|^{2}<\alpha\right)$, $P_{\left.i t|| \mathbf{s}_{i}^{*} \mathbf{s}_{t}\right|^{2} \geq \alpha}=P\left(C\left(\mathbf{s}_{i}\right) \geq \mathcal{C}\left(\mathbf{s}_{t}\right) \mid \mathbf{s}_{t}\right.$ is sent, $\left.\left|\mathbf{s}_{i}^{*} \mathbf{s}_{t}\right|^{2} \geq \alpha\right)$,

$$
\begin{aligned}
& P_{\left.i t|| \mathbf{s}_{i}^{*} \mathbf{s}_{t}\right|^{2}<\alpha}=P\left(\operatorname{Tr}\left(X^{*} \mathbf{s}_{i}\right)\left(X^{*} \mathbf{s}_{i}\right)^{*} \geq\right. \\
&\left.\alpha \operatorname{Tr}\left(X^{*} \mathbf{s}_{t}\right)\left(X^{*} \mathbf{s}_{t}\right)^{*} \mid \mathbf{s}_{t} \text { is sent }\right) .
\end{aligned}
$$

Since we assume that $\mathbf{s}_{t}$ was transmitted, it holds that $X=$ $\sqrt{k} \mathbf{s}_{t} \mathbf{h}+W$ where as earlier $k=\rho T$. Replacing this value for $X$ in (18), we obtain

$$
P_{\left.i t|| \mathbf{s}_{i}^{*} \mathbf{s}_{t}\right|^{2}<\alpha}=P\left(\operatorname{Tr}\left(\left[\begin{array}{c}
\mathbf{h} \\
W
\end{array}\right]^{*} Q_{n}\left[\begin{array}{c}
\mathbf{h} \\
W
\end{array}\right] \geq 0 \mid \mathbf{s}_{t} \text { is sent }\right),\right.
$$

where

$$
\begin{aligned}
& Q_{n}=\left[\begin{array}{c}
\sqrt{k} \mathbf{s}_{t}^{*} \\
I
\end{array}\right]\left(\mathbf{s}_{i} \mathbf{s}_{i}^{*}-\alpha \mathbf{s}_{t} \mathbf{s}_{t}^{*}\right)\left[\begin{array}{ll}
\sqrt{k} \mathbf{s}_{t} & I
\end{array}\right] \\
& =\left[\begin{array}{c}
\sqrt{k} \mathbf{s}_{t}^{*} \\
I
\end{array}\right]\left[\begin{array}{ll}
\mathbf{s}_{i} & \mathbf{s}_{t}
\end{array}\right]\left[\begin{array}{cc}
1 & 0 \\
0 & -\alpha
\end{array}\right]\left[\begin{array}{l}
\mathbf{s}_{i}^{*} \\
\mathbf{s}_{t}^{*}
\end{array}\right]\left[\begin{array}{ll}
\sqrt{k} \mathbf{s}_{t} & I
\end{array}\right] \\
& =\left[\begin{array}{cc}
\sqrt{k} \psi_{i t}^{*} & \sqrt{k} \\
\mathbf{s}_{i} & \mathbf{s}_{t}
\end{array}\right]\left[\begin{array}{cc}
1 & 0 \\
0 & -\alpha
\end{array}\right]\left[\begin{array}{cc}
\sqrt{k} \psi_{i t} & \mathbf{s}_{i}^{*} \\
\sqrt{k} & \mathbf{s}_{i}^{*}
\end{array}\right] \text {, }
\end{aligned}
$$

and $\psi_{i t}=\mathbf{s}_{i}^{*} \mathbf{s}_{t}$. Although it is possible to compute explicitly the probability in (19), we will find that it is sufficient to find its Chernoff bound. In particular,

$$
\begin{aligned}
& P_{\left.i t|| \mathbf{s}_{i}^{*} \mathbf{s}_{t}\right|^{2}<\alpha} \leq \min _{\mu} E e^{\mu\left(\operatorname{Tr}\left(\left[\begin{array}{c}
\mathbf{h} \\
W
\end{array}\right]^{*} Q_{n}\left[\begin{array}{c}
\mathbf{h} \\
W
\end{array}\right]\right)\right)}=
\end{aligned}
$$

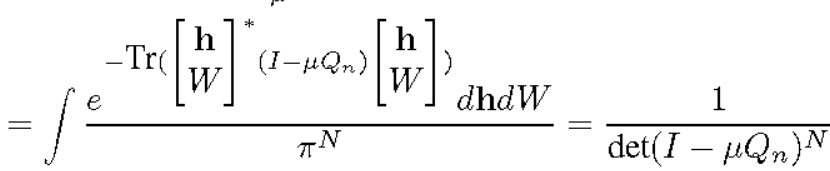

We first simplify the determinant in the denominator as

$$
\operatorname{det}\left(I-\mu Q_{n}\right)=\operatorname{det}\left(I-\mu\left[\begin{array}{cc}
k \psi_{i t} \psi_{i t}^{*}+1 & (k+1) \psi_{i t} \\
-\alpha(k+1) \psi_{i t}^{*} & -\alpha(k+1) 1
\end{array}\right]\right)
$$

After some further algebraic transformations we obtain

$\operatorname{det}\left(I-\mu Q_{n}\right)=(k+1) \alpha\left(V^{(i t)}-1\right)\left(-\mu+\xi^{(1)}\right)\left(-\mu+\xi^{(2)}\right)$

with

$$
\begin{aligned}
& \xi^{(1)}=\frac{V^{(i t)}-\alpha+\frac{1-\alpha}{k}+\sqrt{\left(V^{(i t)}-\alpha+\frac{1-\alpha}{k}\right)^{2}+\frac{4 \alpha\left(1-V^{(i t)}\right)(k+1)}{k^{2}}}}{2 \alpha\left(V^{(i t)}-1\right) \frac{k+1}{k}}, \\
& \xi^{(2)}=\frac{V^{(i t)}-\alpha+\frac{1-\alpha}{k}-\sqrt{\left(V^{(i t)}-\alpha+\frac{1-\alpha}{k}\right)^{2}+\frac{4 \alpha\left(1-V^{(i t)}\right)(k+1)}{k^{2}}}}{2 \alpha\left(V^{(i t)}-1\right) \frac{k+1}{k}},
\end{aligned}
$$

and $V^{(i t)}=\psi_{i t} \psi_{i t}^{*}$. Although our results will hold for any SNR, to make writing less tedious in the rest of the paper we consider only the case of large SNR. Therefore, the previous results simplify to

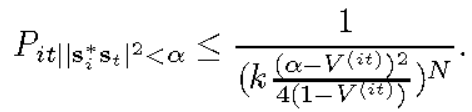

To compute the bound on $P\left(C\left(\mathbf{s}_{i}\right) \geq\right.$ $\mathcal{C}\left(\mathbf{s}_{t}\right) \mid \mathbf{s}_{t}$ is sent, $\left.\left|\mathbf{s}_{i}^{*} \mathbf{s}_{t}\right|^{2} \geq \alpha\right)$ we will use a well known result from the literature (see e.g.,[7])

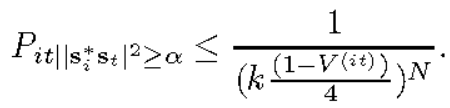

Now we can substitute the results from (20) and (21) in (17) and obtain

$$
\begin{aligned}
& P_{g}\left(\text { error } \mid \mathbf{s}_{t} \text { is sent }\right) \leq \sum_{\left|\mathbf{s}_{i}^{*} \mathbf{s}_{t}\right|^{2}<\alpha} 2 \frac{1}{\left(k \frac{\left(\alpha-V^{(i t)}\right)^{2}}{4\left(1-V^{(i t}\right)}\right)^{N}} \\
&+\sum_{\left|\mathbf{s}_{i}^{*} \mathbf{s}_{t}\right|^{2} \geq \alpha} \frac{1}{\left(k \frac{\left(1-V^{(i t)}\right)}{4}\right)^{N}}=B^{p e p}(\rho) .
\end{aligned}
$$

Recall that in the case of the exact ML decoding, which requires algorithms none of which is of polynomial complexity, we have for the same probability of error

$$
\begin{aligned}
& P_{M L}\left(\text { error } \mid \mathbf{s}_{t} \text { is sent }\right) \leq \sum_{\left|\mathbf{s}_{i}^{*} \mathbf{s}_{t}\right|^{2}<\alpha} \frac{1}{\left(k \frac{\left(1-V^{(i t)}\right)}{4}\right)^{N}} \\
&+\sum_{\left|\mathbf{s}_{i}^{*} \mathbf{s}_{t}\right|^{2} \geq \alpha} \frac{1}{\left(k \frac{\left(1-V^{(i t)}\right)}{4}\right)^{N}}=B_{M L}^{p e p}(\rho) .
\end{aligned}
$$

Clearly, comparing (22) and (23) it follows that the algorithm based on the well known SDP relaxation (slightly refined here for the purposes of the valid proof) has the same diversity as the exact ML solution. Of course, since the SDP-relaxation algorithm is only an approximation, the exact ML solution still has an advantage of $\left(\frac{1-V^{(i t)}}{\alpha-V^{(i t)}}\right)^{2}$ in the coding gain. It should also be noted that a very similar result related to the diversity of the SDP-based algorithm in the context of coherent (channel known at the receiver) ML-detection has recently been shown in [5].

We summarize the previous results in the following theorem.

Theorem 1: Consider the problem of non-coherent MLdetection for a SIMO system described in (1). Assume that the codeword $s_{t}$ was transmitted. Then the probability that an error occurred if Algorithm 1 was applied to solve (3), can be upper bounded in the following way

$$
\begin{aligned}
P\left(\text { error } \mid \mathbf{s}_{t} \text { is sent }\right) & \leq \sum_{\left|\mathbf{s}_{i}^{*} \mathbf{s}_{t}\right|^{2}<\alpha} 2 \frac{1}{\left(\rho T \frac{\left(\alpha-V^{(i t)}\right)^{2}}{4\left(1-V^{(i t)}\right)}\right)^{N}} \\
& +\sum_{\left|\mathbf{s}_{i}^{*} \mathbf{s}_{t}\right|^{2} \geq \alpha} \frac{1}{\left(\rho T \frac{\left(1-V^{(i t)}\right)}{4}\right)^{N}} .
\end{aligned}
$$

Proof: Follows from the previous discussion

\section{ASYMPTOTIC ANALYSIS, $T \rightarrow \infty$}

In this section we explicitly compute the ratio of the bounds on the probability of error ( $P$ (error $\mid \mathrm{s}_{t}$ is sent) and $P_{M L}$ (error $\mid \mathbf{s}_{t}$ is sent $)$ ) in the case when $T \rightarrow \infty$. We first explicitly analyze a special case $q=2$ which correspond to 2-PSK. Afterwards we derive the corresponding results for general q-PSK.

\section{A. $q=2$}

In this section we will compute in the limit of large $T$ the following quantity

$$
K_{\alpha}=\sum_{\left|\mathbf{s}_{i}^{*} \mathbf{s}_{t}\right|^{2}<\alpha} \frac{1}{\left(\frac{\left(\alpha-V^{(i t)}\right)^{2}}{\left(1-V^{(i t)}\right)}\right)^{N}} .
$$

Let $x_{\min }=\left\lfloor\frac{T(1-\sqrt{\alpha})}{2}\right\rfloor$. Then it is not difficult to see that 


$$
K_{\alpha}=\sum_{x=x_{\min }+1}^{T-\left(x_{\min }+1\right)}\left(\begin{array}{l}
T \\
x
\end{array}\right) \frac{1}{\left(\frac{\left(\alpha-\left(\frac{T-2 x}{T}\right)^{2}\right)^{2}}{\left(1-\left(\frac{T-2 x}{T}\right)^{2}\right)}\right)} .
$$

Before proceeding further let us examine more carefully the behavior of $\left(\begin{array}{l}T \\ x\end{array}\right)$ when $T$ is large. First let $x=p_{1} T$. Then we have that $\left(\begin{array}{l}T \\ x\end{array}\right)=\frac{T !}{\left(p_{1} T\right) !\left(p_{2} T\right) !}$, where $p_{1}+p_{2}=1$. Furthermore we have that

$$
\ln T ! \approx\left(T+\frac{1}{2}\right) \ln T-T+\frac{1}{2} \ln (2 \pi)
$$

and similarly

$\ln \left(p_{i} T\right) ! \approx p_{i} T \ln T+\frac{1}{2} \ln T+p_{i} T \ln p_{i}+\frac{1}{2} \ln p_{i}-p_{i} T+\frac{1}{2} \ln (2 \pi)$

Combining (26) and (27) we obtain

$$
\left(\begin{array}{c}
T \\
x
\end{array}\right)=\frac{T !}{\left(p_{1} T\right) !\left(p_{2} T\right) !} \approx \frac{e^{T H\left(p_{1}\right)}}{\sqrt{2 T \pi p_{1}\left(1-p_{1}\right)}}
$$

where $H\left(p_{1}\right)=-p_{1} \ln p_{1}-\left(1-p_{1}\right) \ln \left(1-p_{1}\right)$ is the entropy function. Replacing (28) in (25) and assuming $T \rightarrow \infty$ we further have

$$
\begin{aligned}
& K_{\alpha} \approx \sum_{x=\left(x_{\min }+1\right)}^{T-\left(x_{\min }+1\right)} \frac{e^{T H(x / T)}}{\sqrt{2 \pi x(1-x / T)}} \frac{1}{\left(\frac{\left(\alpha-(1-2 x / T)^{2}\right)^{2}}{1-(1-2 x / T)^{2}}\right)^{N}} \\
& \approx \int_{\left(x_{\min }+1\right) / T}^{1-\left(x_{\min }+1\right) / T} T \frac{e^{T H\left(p_{1}\right)}}{\sqrt{2 T \pi p_{1}\left(1-p_{1}\right)}} \frac{d p_{1}}{\left(\frac{\left(\alpha-\left(1-2 p_{1}\right)^{2}\right)^{2}}{1-\left(1-2 p_{1}\right)^{2}}\right)^{N}} .
\end{aligned}
$$

To solve the previous integral we will use the saddle point method. Let $g\left(p_{1}\right)=\frac{T}{\sqrt{2 T \pi p_{1}\left(1-p_{1}\right)}\left(\frac{\left(\alpha-\left(1-2 p_{1}\right)^{2}\right)^{2}}{1-\left(1-2 p_{1}\right)^{2}}\right)^{N}}$. The saddle point method gives

$$
K_{\alpha} \approx e^{T H\left(p_{0}\right)} g\left(p_{0}\right) \sqrt{\frac{2 \pi}{\left.T \frac{d^{2} H\left(p_{1}\right)}{d p_{1}^{2}}\right|_{p_{1}=p_{0}}}}
$$

where $p_{0}$ is solution to $\frac{d H\left(p_{1}\right)}{d p_{1}}=0$. Then it easily follows $p_{0}=$ $\frac{1}{2}, H\left(p_{0}\right)=\ln 2, g\left(p_{0}\right)=\frac{T}{\sqrt{T \pi / 2} \alpha^{2 N}}$, and $\left.\frac{d^{2} H\left(p_{1}\right)}{d p_{1}^{2}}\right|_{p_{1}=p_{0}}=4$. Using all of this (30) becomes

$$
K_{\alpha} \approx \frac{2^{T} T}{\sqrt{T \pi / 2} \alpha^{2 N}} \sqrt{\frac{1}{2 T}}=\frac{2^{T}}{\alpha^{2 N}} .
$$

We summarize the previous analysis in the following theorem.

Theorem 2: Consider the problem of non-coherent MLdetection for a SIMO system described in (1). Assume that the elements of the transmitted codeword $s_{t}$ are chosen from 2-BSK constellation and that $T \rightarrow \infty$. Let $B^{p e p}(\rho)$ defined in (22) be the PEP type bound on the probability that an error occurred if Algorithm 1 was applied to solve (3). Let $B_{M L}^{p e p}$ defined in (23) be the PEP type bound on the probability that an error occurred if an exact ML algorithm was applied to solve (3). Then $B^{p e p}(\rho)$ and $B_{M L}^{p e p}(\rho)$ differ at most by 3.92 dB, i.e. $B^{\text {pep }}\left(\rho / \alpha^{2}\right) \leq B_{M L}^{p e p}(\rho)$ and $10 \log \frac{1}{\alpha^{2}}=3.92 \mathrm{~dB}$.

Proof: The fact that if $q=2$ then $\alpha=\frac{2}{\pi}$ was proved in [2]. The rest follows by combining (22), (23), and (31).

\section{B. General q}

In this subsection we generalize the result for 2-PSK to qPSK. In q-PSK case the elements of a vector $\mathrm{s}_{i}$ are from the set $\mathcal{Z}=\left\{\frac{1}{\sqrt{T}}, \frac{e^{\frac{j 2 \pi}{q}}}{\sqrt{T}}, \frac{e^{\frac{j 4 \pi}{q}}}{\sqrt{T}}, \ldots, \frac{e^{\frac{j 2(q-1) \pi}{q}}}{\sqrt{T}}\right\}$ and as shown in [3] and [4] $\alpha=\frac{(q \sin (\pi / q))^{2}}{4 \pi}$. As in the previous subsection let $z_{l}=s_{i_{b}}^{*} s_{t_{l}}$ and $\mathbf{z}=\left[z_{1}, z_{2}, \ldots, z_{T}\right]$. Clearly the elements of $\mathbf{z}$ are also from the set $\mathcal{Z}$. Let $x_{1}, x_{2}, \ldots, x_{q}$ be the numbers of elements in $\mathbf{z}$ that are equal to $\frac{1}{T}, \frac{e^{\frac{2 \pi}{q}}}{T}, \frac{e^{\frac{j 4 \pi}{q}}}{T}, \ldots, \frac{e^{\frac{j 2(q-1) \pi}{q}}}{T}$, respectively. Then it easily follows that

$$
\begin{gathered}
\left|\mathbf{s}_{i}^{*} \mathbf{s}_{t}\right|^{2}=\left[\left(\left(T-\sum_{i=1}^{q-1} x_{i}\right) \cos \left(\frac{2(q-1) \pi}{q}\right)+\sum_{i=1}^{q-1} x_{i} \cos \left(\frac{2(i-1) \pi}{q}\right)\right)^{2}\right. \\
\left.+\left(\left(T-\sum_{i=1}^{q-1} x_{i}\right) \sin \left(\frac{2(q-1) \pi}{q}\right)+\sum_{i=1}^{q-1} x_{i} \sin \left(\frac{2(i-1) \pi}{q}\right)\right)^{2}\right] / T^{2} \\
\text { Let } p_{i}=x_{i} / T, 1 \leq i \leq q, \mathbf{p}=\left[p_{1}, p_{2}, \ldots, p_{q-1}\right], \text { and } \\
f(\mathbf{p})=\left(\left(1-\sum_{i=1}^{q-1} p_{i}\right) \cos \left(\frac{2(q-1) \pi}{q}\right)+\sum_{i=1}^{q-1} p_{i} \cos \left(\frac{2(i-1) \pi}{q}\right)\right)^{2} \\
+\left(\left(T-\sum_{i=1}^{q-1} p_{i}\right) \sin \left(\frac{2(q-1) \pi}{q}\right)+\sum_{i=1}^{q-1} p_{i} \sin \left(\frac{2(i-1) \pi}{q}\right)\right)^{2}
\end{gathered}
$$

Then similarly to (25) we have

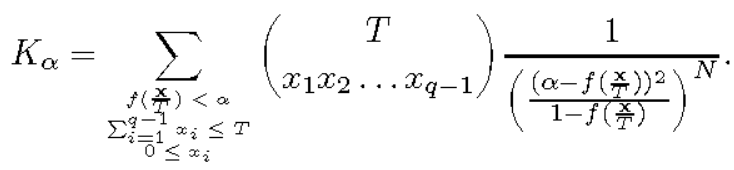

It is not difficult to see that (28) can be generalized in the following way

$$
\begin{aligned}
& \left(\begin{array}{c}
T \\
x_{1} x_{2} \ldots x_{q-1}
\end{array}\right)=\frac{T !}{\left(p_{1} T\right) !\left(p_{2} T\right) ! \ldots\left(p_{q} T\right) !} \\
& \quad \approx \frac{e^{T H(\mathrm{p})}}{\left(2 T \pi\left(1-\sum_{i}^{q-1} p_{i}\right) \prod_{i=1}^{q-1} p_{i}\right)^{(q-1) / 2}} .
\end{aligned}
$$

Then similarly to (29) replacing (33) in (32) we have

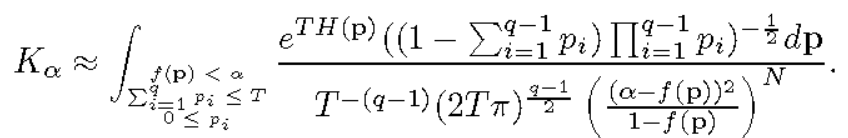

Let $\mathbf{p}_{0}=[1 / q, 1 / q, \ldots, 1 / q]$ be the solution of $\frac{d H(\mathrm{p})}{d \mathrm{p}}=0$ and let $\mathcal{H}(H(\mathbf{p}))$ be the Hessian of $H(\mathbf{p})$. Further let $g(\mathbf{p})=$ $\frac{\left(\left(1-\sum_{i=1}^{q-1} p_{i}\right) \prod_{i=1}^{q-1} p_{i}\right)^{-\frac{1}{2}}}{T^{-(q-1)}(2 T \pi)^{\frac{g-1}{2}}\left(\frac{(\alpha-f(\mathbf{p}))^{2}}{1-f(\mathbf{p})}\right)^{N}}$. Then since entropy is a convex function we can similarly to $(30)$ write

$$
K_{\alpha} \approx e^{T H\left(\mathbf{p}_{0}\right)} g\left(\mathbf{p}_{0}\right)\left(\frac{2 \pi}{T\left(\left.\operatorname{det} \mathcal{H}(H(\mathbf{p}))\right|_{\mathbf{p}=\mathbf{p}_{0}}\right)^{1 /(q-1)}}\right)^{\frac{q-1}{2}} .
$$

It is easy to see that $H\left(\mathbf{p}_{0}\right)=\ln q,\left.\operatorname{det} \mathcal{H}(H(\mathbf{p}))\right|_{\mathbf{p}=\mathrm{p}_{0}}=q^{q}$, $h\left(\mathbf{p}_{0}\right)=0$, and $g\left(\mathbf{p}_{0}\right)=\frac{T^{q-1}\left(q^{q}\right)^{\frac{1}{2}}}{(2 T \pi)^{\frac{q-1}{2}} \alpha^{2 N}}$. Replacing these values in (34) we finally obtain

$$
K_{\alpha} \approx e^{T \ln q} \frac{T^{q-1}\left(q^{q}\right)^{\frac{1}{2}}}{(2 T \pi)^{\frac{q-1}{2}} \alpha^{2 N}}\left(\frac{2 \pi}{T\left(q^{q}\right)^{\frac{1}{q-1}}}\right)^{\frac{q-1}{2}}=\frac{q^{T}}{\alpha^{2 N}} .
$$


We summarize the previous analysis in the following theorem.

Theorem 3: Consider the problem of non-coherent MLdetection for a SIMO system described in (1). Assume that the elements of the transmitted codeword $s_{t}$ are chosen from q-BSK $(q \geq 4)$ constellation and that $T \rightarrow \infty$. Let $B^{p e p}(\rho)$ defined in (22) be the PEP type upper bound on the probability that an error occurred if Algorithm 1 was applied to solve (3). Let $B_{M L}^{p e p}$ defined in (23) be the PEP type upper bound on the probability that an error occurred if an exact ML algorithm was applied to solve (3). Then

$$
B^{p e p}\left(\rho / \alpha^{2}\right) \leq B_{M L}^{p e p}(\rho) .
$$

Furthermore, assume that there are two SNR's $\rho_{S D P}$ and $\rho_{M L}$ such that $B^{p e p}\left(\rho_{S D P}\right)=B_{M L}^{p e p}\left(\rho_{M L}\right)$. Then it holds

$$
\Delta \rho(q)=\rho_{S D P}-\rho_{M L} \leq 20 \log \left(\frac{4 \pi}{(q \sin (\pi / q))^{2}}\right)
$$

dB.

We further have $\Delta \rho(4)=3.92, \Delta \rho(8)=2.547 \mathrm{~dB}, \Delta \rho(16)=$ $2.21 \mathrm{~dB}$, and

$$
\lim _{q \rightarrow \infty} \Delta \rho(q)=2.0982 \mathrm{~dB} .
$$

Proof: Follows by combining (22), (23), and (35).

Effectively, Theorem 3 states that if a codeword was transmitted then its averaged (averaging is over all other codewords) pairwise probabilities of error in the case of exact ML and approximate SDP detection differ by at most $\Delta \rho(q) \mathrm{dB}$.

\section{Computational COMPLEXity}

At the end, let us elaborate briefly on the complexity of the algorithm that we proposed. By carefully inspecting it, one can note that due to the modification of the conventional SDP randomized algorithm, our algorithm, is strictly speaking, no longer polynomial. However, for most practical cases the additional amount of operations on top of the basic SDP core of the algorithm is of effectively negligible complexity. To examine this let us study the case of 2 and 4-PSK (for q-PSK similar arguments can be established). Note that the additional complexity is equal to the number of the vectors $\mathbf{s},\left|S^{c}\right|$, which satisfy inequality $\left|\mathbf{s}^{*} \hat{\mathbf{s}}\right|^{2} \geq \alpha=\frac{2}{\pi}$. Clearly, in the case of 2PSK this number can be upper-bounded as

$$
\left|S^{c}\right| \leq\left\lfloor\frac{T(1-\sqrt{\alpha})}{2}\right\rfloor\left(\begin{array}{c}
T \\
\left\lfloor\frac{T(1-\sqrt{\alpha})}{2}\right\rfloor
\end{array}\right) \leq T^{4.2} \text {, if } T<60,
$$

where we have assumed that for $T<60$ the complexity of solving an SDP is $60^{4.2}$. In 4-PSK case we can numerically obtain

$$
\left|S^{c}\right| \leq \sum_{f\left(\frac{x}{T}\right) \geq \alpha, 0 \leq x_{i} \leq T, x_{1} \neq T}\left(\begin{array}{c}
T \\
x_{1} x_{2} x_{3}
\end{array}\right) \leq T^{4.71} \leq T^{4.8}, T \leq 24
$$

where we have assumed that complexity of solving an SDP of dimension $T \leq 24$ is at least $T^{4.8}$.

However, for large $T,\left(\begin{array}{c}T \\ x_{\min }\end{array}\right) \approx \frac{2^{T H\left(x_{\min } / T\right)}}{\sqrt{2 \pi x_{\min }\left(1-x_{\min } / T\right)}}$, (where $H$ is the entropy function) and one can show that

$$
\left|S^{c}\right| \geq\left(\begin{array}{c}
T \\
\left\lfloor\frac{T(1-\sqrt{\alpha})}{2}\right\rfloor
\end{array}\right) \approx \frac{2^{T H(\lfloor(1-\sqrt{\alpha}) / 2\rfloor)}}{\sqrt{2 \pi x_{\min }\left(1-\frac{x_{\min }}{T}\right)}}=\frac{2^{0.47 T}}{\sqrt{0.1817 \pi T}} .
$$

The previous expression implies that the additional amount of computation introduced to ensure the validity of our proof is indeed exponential, while of course in the limit of large $T$ the complexity of solving SDP becomes $T^{3.5}$. However, the exponential constant is two times smaller than in the exhaustive search. Therefore, in communications, where the dimension of SIMO systems is smaller than 60 and 2-PSK signalling is used or where the dimension of SIMO system is smaller than 24 and 4-PSK signalling is used, the complexity of our algorithm is of the same order as the complexity of the SDP.

\section{DisCUSSION AND CONCLUSION}

We proposed a modification of the SDP relaxation for solving the non-coherent ML detection problem in singleinput multiple-output communication systems with q-PSK signalling. The computed PEP implies that the performance of the algorithm is comparable to that of the optimal ML solution, but is obtained at potentially significantly lower computational complexity. Of course, it would be of a great interest if one could construct a provably polynomial time algorithm which has the same PEP performance as the one we analyzed in this paper. That will be the subject of a future work.

\section{ACKNOWLEDGMENT}

This work was supported in part by the National Science Foundation under grant no. CCR-0133818, by the David and Lucille Packard Foundation, and by Caltech's Lee Center for Advanced Networking.

\section{REFERENCES}

[1] M. Goemans and D. Williamson, "Improved approximation algorithms for maximum cut and satisfiability problems using semidefinite programming," Journal of the ACM, 42(6):1115-1145 1995.

[2] Y. Nesterov, "Quality of semidefinite relaxation for nonconvex quadratic optimization" CORE discussion paper, CORE, March 1997.

[3] A.M. So, J. Zhang, Y. Ye, "On Approximating Complex Quadratic Optimization Problems via Semidefinite Programming Relaxations," to appear in Math Programming.

[4] S. Zhang and Y. Huang, "Complex Quadratic Optimization and Semidefinite Programming," SIAM Joumal on Optimization, 16(3):871-890, 2006

[5] J. Jalden and B. Ottersten, "The diversity order of the semidefinite relaxation detector," submitted to IEEE Transactions on Information Theory, 2006, (availiable at http://arxiv.org/abs/cs/0606083).

[6] L. Engbresten, P. Indyk and R. O'Donnell, "Derandomized dimensionality reduction with application," 13th Symposium on Discrete Algorithms, 2002.

[7] B. Hochwald and T. Marzetta, "Unitary space time modulation for multiantenna modulation in Rayleigh flat fading channel," IEEE Trans. on Inf. Theory, March. 2000.

[8] H. Vikalo, B. Hassibi and P. Stoica, "Joint ML estimation and signal detection," IEEE Trans. on Wireless Communication, July 2006.

[9] W.K. Ma, T.N. Davidson, K.M. Wong, Z.-Q. Luo and P.C. Ching, "Quasimaximum-likelihood multiuser detection using semi-definite relaxation," IEEE Transactions on Signal Processing, vol. 50, no. 4, pp. 912-922, 2002.

[10] M. Kisialiou and Z.-Q. Luo, "Performance Analysis of Quasi-MaximumLikelihood Detector Based on Semi-Definite Programming," Proc. of ICASSP, pp. 433-436, Philadelphia, PA, March 2005.

[11] M.Stojnic, B. Hassibi, and H. Vikalo, "PEP analysis of the SDP-based joint channel estimation and signal detection," to be presented at ICASSP International Conference on Acoustics, Signal and Speech Processing 2007. 